\title{
Gross tumor volume is an independent prognostic factor in patients with postoperative locoregional recurrence of esophageal squamous cell carcinoma
}

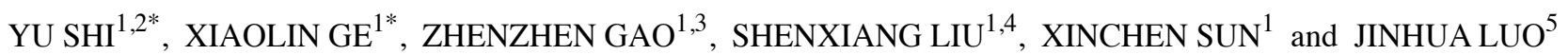 \\ ${ }^{1}$ Department of Radiotherapy, The First Affiliated Hospital of Nanjing Medical University, Nanjing, Jiangsu 210029; \\ ${ }^{2}$ Department of Radiotherapy, Affiliated Hospital of Nantong University, Nantong, Jiangsu 226001; ${ }^{3}$ Department of Oncology, \\ The Second Affiliated Hospital of Jiaxing University, Jiaxing, Zhejiang 314000; ${ }^{4}$ Department of Oncology, \\ Affiliated Hospital of Yangzhou University, Yangzhou, Jiangsu 225100; ${ }^{5}$ Department of Thoracic Surgery, \\ The First Affiliated Hospital of Nanjing Medical University, Nanjing, Jiangsu 210029, P.R. China
}

Received April 15, 2019; Accepted June 10, 2019

DOI: $10.3892 / 01.2019 .10529$

\begin{abstract}
Many cases of esophageal squamous cell carcinoma (ESCC) involve lymph node and distant metastases after esophagectomy, and most patients relapse within 2 years. Intensity-modulated radiotherapy (IMRT) is an important treatment for these cases of recurrence in ESCC and is widely used in clinical practice. A retrospective study of 137 postoperative patients with locoregional recurrences of ESCC who received IMRT was carried out. Kaplan-Meier survival curves and log-rank tests of univariate analysis was performed to assess whether there was a significant association between demographic and clinical features and death after recurrence. For multivariate analysis, the statistically significant results from the Kaplan-Meier method were subjected to Cox regression analysis. A total of 109 male and 28 female patients were included. There were $21(15.3 \%)$, $58(42.3 \%), 36(26.3 \%), 3(2.2 \%), 17(12.4 \%)$, and $2(1.5 \%)$ recurrences in the anastomotic, supraclavicular, mediastinal, tumor bed, polyregional, and abdominal regions, respectively. Univariate analysis showed that the gross tumor volume (GTV) of radiation $\left(<27 \mathrm{vs} . \geq 27 \mathrm{~cm}^{3}\right)$ and the number of lymph nodes were significantly associated with
\end{abstract}

Correspondence to: Dr Xinchen Sun, Department of Radiotherapy, The First Affiliated Hospital of Nanjing Medical University, 300 Guangzhou Road, Nanjing, Jiangsu 210029, P.R. China

E-mail: rvw64v@163.com; sunxinchen2012@163.com

Dr Jinhua Luo, Department of Thoracic Surgery, The First Affiliated Hospital of Nanjing Medical University, 300 Guangzhou Road, Nanjing, Jiangsu 210029, P.R. China

E-mail: ljhua1966@126.com

\section{*Contributed equally}

Key words: esophageal squamous cell carcinoma, prognostic factors, radiation therapy survival. The survival rates of patients at 1,2, 3 and 5 years with $\mathrm{GTV}<27 \mathrm{~cm}^{3}$ were $72.7,51.5,37.1$ and $25.9 \%$, respectively, and with GTV $\geq 27 \mathrm{~cm}^{3}$ were $63.7,26.9,17.9$ and $0 \%$, respectively. The significant independent prognostic factor was GTV $\left[<27\right.$ vs. $\geq 27 \mathrm{~cm}^{3}$; hazard ratio (HR), 1.746; $95 \%$ confidence interval (CI), 1.112-2.741]. In conclusion, GTV of radiation $\left(<27\right.$ vs. $\left.\geq 27 \mathrm{~cm}^{3}\right)$ is an independent factor in predicting locoregional recurrence after ESCC. Patients with $\mathrm{GTV}<27 \mathrm{~cm}^{3}$ are likely to have a better prognosis.

\section{Introduction}

Esophageal carcinoma (EC) is one of the most fatal and prevalent human malignancies worldwide, that due to its rapidly increasing incidence, it has gained worldwide attention (1). Esophageal squamous cell carcinoma (ESCC) is the most lethal pathological type, accounting for $\sim 90 \%$ of total EC cases, and China alone contributes to more than half of the global ESCC cases (2). At present, surgery is the main treatment for EC, but the 5-year survival rate after simple surgery is $31-55 \%$ (3). Most patients have lymph node involvement and distant metastases after esophagectomy and the 5-year survival rate of ESCC patients with metastasis is only 5-47\% (4-6). Moreover, locoregional recurrence and distant metastasis are the most common cause of treatment failure (7). The most common recurrence sites include the anastomosis and regional lymph nodes, which lead to a relatively poorer prognosis $(8,9)$. The overall 2-year post-recurrence survival rate is $12.6 \%$, and the median postoperative survival is 6 months for patients with postoperative recurrence of ESCC (10). Significant difficulty is often encountered in identifying the appropriate treatment method from the limited available treatment options for recurrence following resection.

Recent advances in radiation techniques and chemotherapy may lead to the improvement of the treatment outcomes of postoperatively recurrent ESCC. Three-dimensional conformal radiotherapy (3D-CRT)-based concurrent chemoradiotherapy (CCRT) has been reportedly effective against the recurrence of ESCC after radical surgery (9). 3D-CRT for 
lymph node stations near ESCC with involved-field irradiation may deliver considerable doses of incidental nodal irradiation and promote the elimination of subclinical lesions (11). Intensity-modulated radiotherapy (IMRT), a more advanced form of 3D-CRT, is a high-precision conformal technique that allows physicians to modulate the intensity of the radiation and apply high-radiation doses to the tumor (12). Postoperative precautionary IMRT has high local control rate and acceptable side-effects on thoracic ESCC (13). In this study, we evaluated the survival of ESCC patients that received IMRT for the treatment of postoperative recurrence and evaluated the prognostic factors affecting survival.

\section{Patients and methods}

Acquisition of clinical data. A total of 137 ESCC patients, who received IMRT for postoperative locoregional recurrence at The First Affiliated Hospital of Nanjing Medical University (Nanjing, China) from August 2003 to January 2018, were retrospectively studied. Locoregional recurrence was defined as anastomotic or lymph node recurrence and was confirmed by enhanced computed tomography (CT) and biopsy under endoscopy, respectively. All patients were diagnosed with ESCC by postoperative pathology and distant metastasis was ruled out by imaging. The study was approved by the Ethics Committee of The First Affiliated Hospital of Nanjing Medical University. Patients who participated in this research had complete clinical data. Signed informed consents were obtained from the patients or their guardians.

Clinical data of postoperative tumor recurrence were collected including sex and age, previous treatment (neoadjuvant therapy, postoperative radiotherapy (PORT), and/or chemotherapy), grades of differentiation, primary esophageal tumor location, TNM staging, number and diameter of metastatic lymph nodes, recurrence sites, CCRT, death from recurrence, gross tumor volume (GTV), and survival time after recurrence.

Treatment and follow-up assessment. IMRT was performed according to the standard program in our Department. Briefly, patients were fixed in position with a thermoplastic immobilizer. For treatment simulation and planning purposes, CT scanning images were obtained to determine the radiation treatment plan and target volume. The $\mathrm{CT}$ scan range included the full mediastinum, lower neck, and upper abdomen, with a layer spacing of $5 \mathrm{~mm}$. The images were transmitted to the IMRT treatment planning system and the attending physician's outline of the GTV was reviewed by the chief physician. The GTV included positive lymph nodes based on CT and positron emission tomography (PET), including swollen lymph nodes identified by physical examination and positive lymph nodes shown by PET-CT. The clinical target volume (CTV) was defined as the GTV plus a $1-1.5 \mathrm{~cm}$ radial margin, including high-risk regional lymph nodes. The planning target volume (PTV) contained a $0.5-\mathrm{cm}$ extension on the basis of the CTV. The reference dose point was located at the central part of the GTV and was optimized using a dose volume histogram with a 95\% isodose curve covering the PTV. The treatment plan was reviewed and confirmed by the chief physician. In this study, the median dose of radiation therapy was $62.2 \mathrm{~Gy}$ (range, 40-80 Gy). The X-ray irradiation energy was $6 \mathrm{MV}$ administered 5 times/week with a test dose of 2 Gy each time. The maximum doses to the endangered organs, the lungs, and spine were $\mathrm{V}_{20} \leq 25 \%, \mathrm{~V}_{30} \leq 20 \%$, and $\leq 45 \mathrm{~Gy}$, respectively.

Intravenous or oral chemotherapy, CCRT, continuous chemoradiotherapy, radiotherapy alone or chemotherapy dose were taken into account according to the patient's age, general condition, Karnofsky Performance Status (KPS) score, treatment tolerance and compliance. Follow-up data included physical examinations, such as clinical evaluations and CT scans every 3 months. Tumor-related deaths were documented.

Statistical analysis. Statistical analysis was performed using IBM SPSS 19.0 software (IBM Corp.). Quantitative data were expressed as the mean \pm standard deviation (SD) or median (interquartile range), while qualitative data were expressed as numbers and percentages. The mean/median survival times with 95\% CIs at different levels were assessed by the Kaplan-Meier method (14). Univariate analyses using Kaplan-Meier survival curves and log-rank tests were performed to assess whether there was a significant association between demographic and clinical features, such as sex, age, postoperative treatment, and degree of differentiation, and death after recurrence. Statistical significance was determined using the conventional $\mathrm{P}<0.05$ criterion. For multivariate analysis, the statistically significant results from the Kaplan-Meier method were subjected to Cox regression analysis (15), which was used to analyze the effect of multiple variables on survival time.

\section{Results}

Patient characteristics. The clinical and pathological characteristics of the patients are presented in Tables I and II. Between 2003 and 2018, a total of 109 male and 28 female patients were included, with an average age of $62.22 \pm 6.56$ years (range, 44-82 years). Ninety-seven patients (70.8\%) were $\geq 60$ years of age. A total of $43.8 \%$ of patients received PORT and/or chemotherapy or neoadjuvant therapy. Based on TNM staging, 38 (27.7\%), $53(38.7 \%)$, and $46(33.6 \%)$ patients had stage I, II, and III ESCC, respectively. The locations of the primary ESCCs removed by radical resection included the upper, middle, and lower thoracic esophagus in 14 (10.2\%), $67(48.9 \%)$, and $56(40.9 \%)$ patients, respectively. There were $21(15.3 \%), 58(42.3 \%), 36(26.3 \%), 3(2.2 \%), 17(12.4 \%)$, and $2(1.5 \%)$ recurrences in the anastomotic, supraclavicular, mediastinal, tumor bed, polyregional, and abdominal regions, respectively. Ninety-four patients underwent CCRT. The median GTV of radiation was $27.06 \mathrm{~cm}^{3}$ with interquartile range $(14.03,70.48) \mathrm{cm}^{3}$. The analysis showed that the average survival time after recurrence was $18.83 \pm 14.71$ months. Of the 137 patients who underwent IMRT, 84 (61.3\%) died. As shown in Table III, the 2-year survival rates of patients with anastomotic, supraclavicular, and mediastinal lymph node recurrence were $36.4,41.4$, and $37.5 \%$, respectively. The 2-year survival rates of patients with 0,1 , and 2-6 lymph node recurrences were $38.9,50.8$, and $19.7 \%$, respectively, and the 2-year survival rates for patients with and without CCRT were 41.2 and $34.9 \%$, respectively.

Univariate analysis showed that the GTV of radiation $\left(<27\right.$ vs. $\geq 27 \mathrm{~cm}^{3}$ ) and the number of lymph nodes were 
Table I. Patient characteristics (qualitative variables).

\begin{tabular}{|c|c|c|}
\hline Characteristics & $\begin{array}{l}\text { No. of } \\
\text { patients }\end{array}$ & $\begin{array}{c}\text { Constituent } \\
\text { ratio }(\%)\end{array}$ \\
\hline \multicolumn{3}{|l|}{ Sex } \\
\hline Males & 109 & 79.6 \\
\hline Females & 28 & 20.4 \\
\hline \multicolumn{3}{|l|}{ Age (years) } \\
\hline$<60$ & 40 & 29.2 \\
\hline$\geq 60$ & 97 & 70.8 \\
\hline \multicolumn{3}{|l|}{ Postoperative therapy } \\
\hline No & 77 & 56.2 \\
\hline Radiotherapy & 11 & 8.0 \\
\hline Chemotherapy & 34 & 24.8 \\
\hline Radiotherapy and chemotherapy & 13 & 9.5 \\
\hline Neoadjuvant therapy & 2 & 1.5 \\
\hline \multicolumn{3}{|l|}{ Differentiation grades } \\
\hline Poor & 56 & 40.9 \\
\hline Moderate & 76 & 55.5 \\
\hline Well & 3 & 2.2 \\
\hline Unknown & 2 & 1.5 \\
\hline \multicolumn{3}{|l|}{ Primary tumor location } \\
\hline Upper & 14 & 10.2 \\
\hline Middle & 67 & 48.9 \\
\hline Lower & 56 & 40.9 \\
\hline \multicolumn{3}{|l|}{ TNM staging } \\
\hline I & 38 & 27.7 \\
\hline II & 53 & 38.7 \\
\hline III & 46 & 33.6 \\
\hline \multicolumn{3}{|l|}{ No. of lymph nodes } \\
\hline None & 24 & 17.5 \\
\hline 1 & 63 & 46.0 \\
\hline $2-6$ & 15 & 10.9 \\
\hline Fusion & 35 & 25.5 \\
\hline \multicolumn{3}{|l|}{ Recurrence sites } \\
\hline Anastomotic & 21 & 15.3 \\
\hline Supraclavicular lymph nodes & 58 & 42.3 \\
\hline Mediastinal lymph nodes & 36 & 26.3 \\
\hline Tumor bed & 3 & 2.2 \\
\hline Polyregional lymph nodes & 17 & 12.4 \\
\hline Abdominal lymph nodes & 2 & 1.5 \\
\hline \multicolumn{3}{|l|}{ CCRT } \\
\hline Yes & 94 & 68.6 \\
\hline No & 43 & 31.4 \\
\hline \multicolumn{3}{|l|}{ Death } \\
\hline Yes & 84 & 61.3 \\
\hline No & 53 & 38.7 \\
\hline
\end{tabular}

CCRT, concurrent chemoradiotherapy.

significantly related to survival. Other factors, such as differentiation grade and recurrence site, were not related to
Table II. Patient characteristics (quantitative variables).

\begin{tabular}{lcc}
\hline Characteristics & Values & Range \\
\hline Age (years) & $62.22 \pm 6.56$ & $44-82$ \\
Lymph node diameter $(\mathrm{cm})$ & $2.73 \pm 1.88$ & $0-7.9$ \\
GTV $\left(\mathrm{cm}^{3}\right)$ & $27.06(14.03,70.48)$ & $1.73-385.6$ \\
Survival time after & $18.83 \pm 14.71$ & $2-81$ \\
recurrence (months) & & \\
\hline
\end{tabular}

Age, lymph node diameter and survival time after recurrence are expressed as the mean $\pm \mathrm{SD}$, and GTV as the median (interquartile range). GTV, gross tumor volume.

survival (Table III, Fig. 1). The 1-, 2-, 3-, and 5-year survival rates of patients with $\mathrm{GTV}<27 \mathrm{~cm}^{3}$ were $72.7,51.5,37.1$, and $25.9 \%$, respectively, and with GTV $\geq 27 \mathrm{~cm}^{3}$ were $63.7,26.9$, 17.9 , and $0 \%$, respectively. Multivariate regression analysis with a Cox model showed that the GTV of radiation was a significant independent prognostic factor and was significantly related to the risk of death after recurrence [HR (95\% CI), 1.746 (1.112-2.741); $\mathrm{P}=0.016$ ] (Table IV). According to the results, the risk of death for patients with $G T V \geq 27 \mathrm{~cm}^{3}$ was 1.746 times that for patients with $\mathrm{GTV}<27 \mathrm{~cm}^{3}$, with a statistically significant difference.

\section{Discussion}

Currently, surgery is the primary treatment for ESCC. However, the overall recurrence rate of ESCC patients after radical resection ranges from 34 to $79 \%$, while the rate of locoregional recurrence is $21-68 \%(16,17)$. Evidence has indicated that the number of lymph nodes involved and the depth of primary tumor invasion may help in evaluating the recurrence risk in ESCC patients following curative surgery (17). EC guidelines for the national comprehensive cancer network (NCCN) suggest that some patients with local recurrence after surgery can tolerate CCRT $(18,19)$. Approximately $28 \%$ of patients with ESCC achieved long-term survival with the use of CRT for lymph node recurrence after curative resection (20). Current radiation techniques using 3D-CRT with enhanced accuracy using daily image guidance have improved the accuracy of irradiation (21). The incidental irradiation doses with involved-field irradiation have significantly impacted the control of micro-metastasis and may contribute to the elimination of subclinical ESCC lesions (11). It has been reported that the tolerance to 3D-CRT combined with chemotherapy is better than that of the simple 3D-CRT , which is a feasible technology and can improve the overall survival (OS) rate of patients with recurrent ESCC mediastinal lymph node metastasis after surgery (22). In the present study, ESCC patients with anastomotic or lymph node recurrence received IMRT or IMRT-based CCRT and the factors affecting their survival were investigated.

Previous studies have indicated that the number and regions of lymph node recurrences after ESCC are also important factors influencing the efficacy of salvage chemoradiotherapy (23). Jingu et al reported that the median OS 
Table III. Kaplan-Meier analysis of risk factors related to death after tumor recurrence.

\begin{tabular}{|c|c|c|c|c|c|c|}
\hline Characteristics & Median (95\% CI) & $\begin{array}{c}\text { P-value } \\
\text { (log-rank) }\end{array}$ & $\begin{array}{l}1 \text {-year } \\
\text { survival } \\
\text { rate }(\%)\end{array}$ & $\begin{array}{l}\text { 2-year } \\
\text { survival } \\
\text { rate }(\%)\end{array}$ & $\begin{array}{l}\text { 3-year } \\
\text { survival } \\
\text { rate }(\%)\end{array}$ & $\begin{array}{l}5 \text {-year } \\
\text { survival } \\
\text { rate }(\%)\end{array}$ \\
\hline Sex & & 0.160 & & & & \\
\hline Male & $28.48(22.13,34.82)$ & & 65.1 & 34.9 & 25.3 & 13.5 \\
\hline Female & $36.85(23.58,50.12)$ & & 68.0 & 57.7 & 43.2 & - \\
\hline Age (years) & & 0.169 & & & & \\
\hline$<60$ & $27.90(17.33,38.48)$ & & 50.2 & 30.4 & - & 15.2 \\
\hline$\geq 60$ & $30.46(24.24,36.68)$ & & 75.5 & 42.6 & 28.2 & - \\
\hline Diameter of nodes $(\mathrm{cm})$ & & 0.184 & & & & \\
\hline$<2.6(\mathrm{n}=66)$ & $29.76(22.52,37.00)$ & & 73.7 & 43.3 & 29.5 & 11.1 \\
\hline$\geq 2.6(\mathrm{n}=71)$ & $29.00(20.96,37.03)$ & & 62.9 & 35.0 & - & 18.2 \\
\hline $\operatorname{GTV}\left(\mathrm{cm}^{3}\right)$ & & 0.013 & & & & \\
\hline$<27(\mathrm{n}=68)$ & $35.76(27.44,44.09)$ & & 72.7 & 51.5 & 37.1 & 25.9 \\
\hline$\geq 27(n=69)$ & $22.48(16.98,27.98)$ & & 63.7 & 26.9 & 17.9 & 0.0 \\
\hline Postoperative therapy & & 0.286 & & & & \\
\hline No & $32.40(25.24,39.55)$ & & 71.0 & 47.1 & 37.7 & 16.2 \\
\hline Radiotherapy & $21.69(16.36,27.02)$ & & - & 35.1 & - & - \\
\hline Chemotherapy & $25.41(15.51,35.31)$ & & 56.8 & 27.1 & 17.0 & - \\
\hline Radiotherapy and chemotherapy & $16.00(10.08,21.92)$ & & 38.5 & - & - & - \\
\hline Grades of differentiation & & 0.689 & & & & \\
\hline Poorly & $30.48(21.75,39.20)$ & & 65.3 & 44.0 & 30.0 & 20.0 \\
\hline Moderately & $29.90(21.93,37.87)$ & & 69.6 & 39.7 & 31.2 & 12.5 \\
\hline Well & $18.67(15.82,21.51)$ & & 66.7 & - & - & - \\
\hline Primary tumor location & & 0.162 & & & & \\
\hline Upper & $29.82(17.60,42.04)$ & & 71.4 & 53.6 & - & - \\
\hline Middle & $30.85(23.18,38.52)$ & & 75.2 & 43.7 & 28.1 & 14.1 \\
\hline Lower & $26.70(18.86,34.55)$ & & 58.9 & 29.1 & - & - \\
\hline TNM staging & & 0.222 & & & & \\
\hline I & $24.64(18.24,31.04)$ & & 71.1 & 37.8 & 21.6 & - \\
\hline II & $32.70(24.67,40.74)$ & & 74.4 & 49.2 & - & 13.1 \\
\hline III & $27.60(17.78,37.43)$ & & 57.7 & 27.4 & - & - \\
\hline Location of lymph nodes & & 0.073 & & & & \\
\hline No & $23.86(16.12,31.59)$ & & 62.5 & 38.9 & 15.6 & - \\
\hline Supraclavicular lymph nodes & $31.55(23.61,39.48)$ & & 69.4 & 40.9 & - & 18.2 \\
\hline Mediastinal lymph nodes & $37.85(24.05,51.66)$ & & 74.0 & 53.2 & 39.9 & - \\
\hline Polyregional lymph nodes & $13.40(8.42,18.39)$ & & 53.3 & - & - & - \\
\hline No. of lymph nodes & & 0.025 & & & & \\
\hline None & $23.86(16.12,31.59)$ & & 62.5 & 38.9 & 15.6 & - \\
\hline 1 & $38.74(29.28,31.59)$ & & 77.3 & 50.8 & 38.1 & - \\
\hline $2-6$ & $25.56(10.70,40.42)$ & & 63.2 & 19.7 & - & - \\
\hline Fusion & $21.66(13.72,29.60)$ & & 54.0 & - & - & 0.0 \\
\hline Recurrence sites & & 0.838 & & & & \\
\hline Anastomotic & $23.25(15.39,31.12)$ & & 61.9 & 36.4 & 14.5 & - \\
\hline Supraclavicular lymph nodes & $32.03(23.82,40.24)$ & & 71.1 & 41.4 & - & 18.4 \\
\hline Mediastinal lymph nodes & $29.44(18.19,40.70)$ & & 58.5 & 37.5 & 28.2 & - \\
\hline Polyregional lymph nodes & $18.45(13.33,23.57)$ & & 70.1 & - & - & - \\
\hline CCRT & & 0.513 & & & & \\
\hline No & $30.63(20.81,40.45)$ & & 55.3 & 34.9 & - & - \\
\hline Yes & $28.78(22.46,35.10)$ & & 74.5 & 41.2 & 28.5 & 7.9 \\
\hline
\end{tabular}

Bold indicates statistical significance. CI, confidence interval; GTV, gross tumor volume; CCRT, concurrent chemoradiotherapy. 

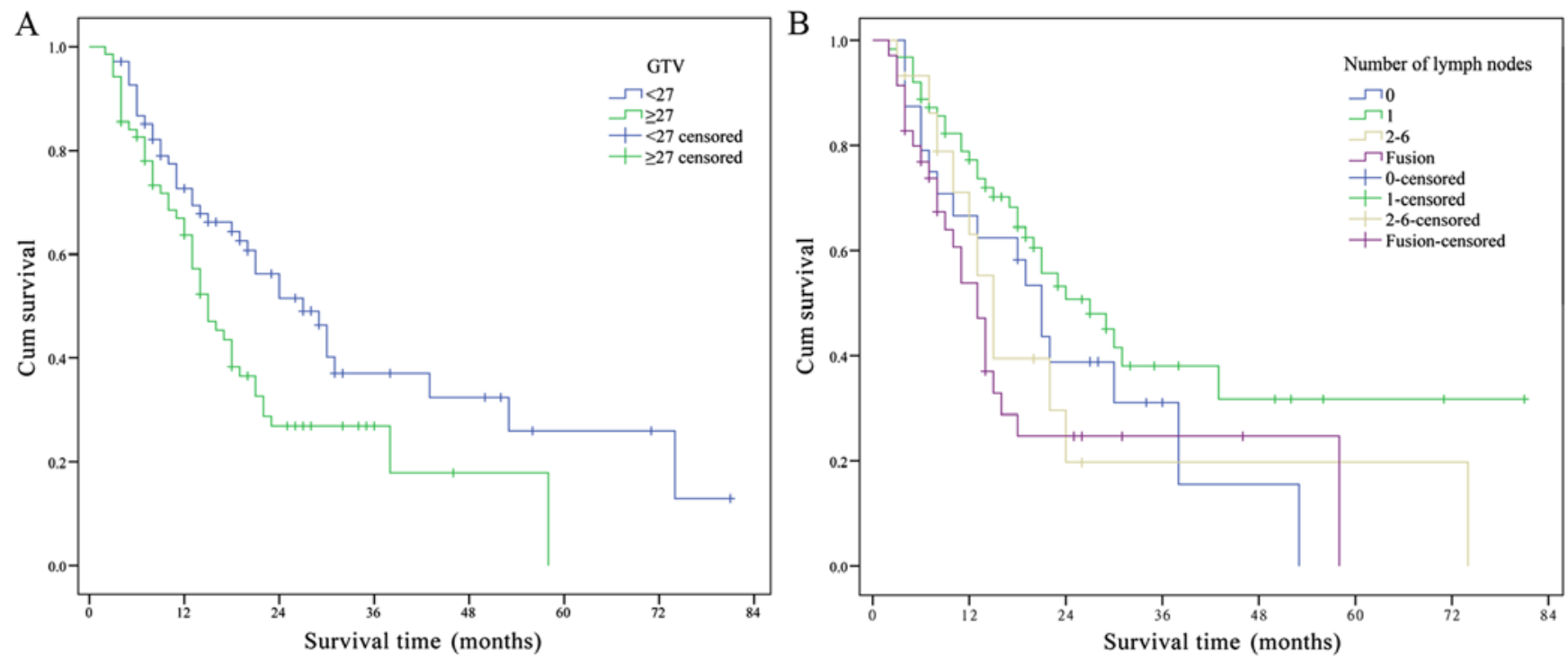

Figure 1. Kaplan-Meier curves estimating the OS of 137 patients with postoperative locoregional recurrence according to (A) the GTV and (B) the number of lymph nodes. OS, overall survival; GTV, gross tumor volume.

Table IV. Multivariate regression model analysis of risk factors related to death after tumor recurrence.

$95.0 \% \mathrm{CI}$ for $\mathrm{HR}$

\begin{tabular}{|c|c|c|c|c|}
\hline \multirow[b]{2}{*}{ Factors } & \multirow[b]{2}{*}{ P-value } & \multirow[b]{2}{*}{ HR } & \\
\hline & & & Lower & Upper \\
\hline GTV & 0.016 & 1.746 & 1.112 & 2.741 \\
\hline No. of lymph nodes & 0.813 & 1.068 & 0.616 & 1.853 \\
\hline
\end{tabular}

CI, confidence interval; HR, hazard ratio; GTV, gross tumor volume.

rates of single and multiple recurrent regions after EC surgery were 39.0 and 6.5 months, respectively, after radiotherapy and chemotherapy, and the number of recurrent lesions was a significant prognostic factor (23). Miyata et al reported that the OS rate of patients with $\geq 4$ recurrent lymph nodes after ESCC surgery was significantly lower than that in patients with $<4$ recurrent lymph nodes (24). Chen et al reported 5-year OS rates for 1-2, 3-6, and $\geq 7$ recurrent lymph nodes after ESCC resection of 33,17 , and $12 \%$, respectively, and patients with more recurrent lymph nodes had worse prognoses (25). In the current study, although Kaplan-Meier analysis showed that the number of lymph nodes was a risk factor related to death after tumor recurrence, multivariate regression model analyses showed that the number of lymph nodes was not an independent prognostic factor.

The risk factor GTV of radiation was a significant independent prognostic factor for survival after recurrence in patients receiving IMRT treatment. The GTVs were contoured as visible tumors on CT scans (26). Most of the lymphatic recurrences after surgery among patients diagnosed with ESCC would have been covered by the proposed PORT CTV (21). The GTV has been demonstrated to be an independent prognostic factor for survival in multiple cancers treated with radiation therapy $(27,28)$. Ma et al reported that the GTV of radiation ( $\geq 5$ vs. $<5 \mathrm{~cm}^{3}$ ) was an important independent prognostic factor in patients with recurrent ESCC (22). However, this study confirmed that GTV of radiation ( $<27$ vs. $\geq 27 \mathrm{~cm}^{3}$ ) is an important independent prognostic factor for the risk of death after recurrence treated by IMRT.

This study has several limitations. Its retrospective design may have a potential bias. In addition, because of the different doses and schedule used in this study, the optimal treatment for locoregional recurrence has not been determined.

In conclusion, our retrospective study showed that the GTV of radiation is an independent prognostic factor in ESCC patients with postoperative locoregional recurrence. Patients with a $G T V \geq 27 \mathrm{~cm}^{3}$ have a higher risk of death.

\section{Acknowledgements}

Not applicable.

\section{Funding}

This study was supported by grants from the National Natural Science Foundation of China (nos. 81703028, 81472809, 81502653 and 81672983 ). We would like to thank everyone involved for their support, and the patients who participated in this study.

\section{Availability of data and materials}

The datasets used and/or analyzed during the present study are available from the corresponding author on reasonable request.

\section{Authors' contributions}

YS and XG performed the retrospective analyses and wrote the manuscript. ZG and SL performed the statistical analysis and revised the manuscript. XS and JL designed the study. All authors read and approved the final manuscript. 


\section{Ethics approval and consent to participate}

The study was approved by the Ethics Committee of The First Affiliated Hospital of Nanjing Medical University (Nanjing, China). Patients who participated in this research had complete clinical data. Signed informed consents were obtained from the patients or their guardians.

\section{Patient consent for publication}

Not applicable.

\section{Competing interests}

The authors declare that they have no competing interests.

\section{References}

1. Siegel RL, Miller KD and Jemal A: Cancer Statistics, 2017. CA Cancer J Clin 67: 7-30, 2017.

2. Zeng H, Zheng R, Zhang S, Zuo T, Xia C, Zou X and Chen W: Esophageal cancer statistics in China, 2011: Estimates based on 177 cancer registries. Thorac Cancer 7: 232-237, 2016.

3. Yao J, Shen X, Li H, Xu J, Shao S, Huang JX and Lin M: LncRNAECM is overexpressed in esophageal squamous cell carcinoma and promotes tumor metastasis. Oncol Lett 16: 3935-3942, 2018

4. Kayani B, Zacharakis E, Ahmed K and Hanna GB: Lymph node metastases and prognosis in oesophageal carcinoma - a systematic review. Eur J Surg Oncol 37: 747-753, 2011.

5. Bai Y, Lin H, Fang Z, Luo Q, Fang Y, Su Y, Hu Q, Duan H, Chen F and Zhang ZY: Plasma microRNA-19a as a potential biomarker for esophageal squamous cell carcinoma diagnosis and prognosis. Biomarkers Med 11: 431-441, 2017.

6. Wu SG, Zhang WW, He ZY, Sun JY, Chen YX and Guo L: Sites of metastasis and overall survival in esophageal cancer: A population-based study. Cancer Manag Res 9: 781-788, 2017.

7. Kato H, Fukuchi M, Miyazaki T, Nakajima M, Kimura H, Faried A, Sohda M, Fukai Y, Masuda N, Manda R, et al: Classification of recurrent esophageal cancer after radical esophagectomy with two- or three-field lymphadenectomy. Anticancer Res 25: 3461-3467, 2005.

8. Doki Y, Ishikawa O, Takachi K, Miyashiro I, Sasaki Y, Ohigashi H, Murata K, Yamada T, Noura S, Eguchi H, et al: Association of the primary tumor location with the site of tumor recurrence after curative resection of thoracic esophageal carcinoma. World J Surg 29: 700-707, 2005.

9. Bao Y, Liu S, Zhou Q, Cai P, Anfossi S, Li Q, Hu Y, Liu M, Fu J, Rong T, et al: Three-dimensional conformal radiotherapy with concurrent chemotherapy for postoperative recurrence of esophageal squamous cell carcinoma: Clinical efficacy and failure pattern. Radiat Oncol 8: 241, 2013.

10. Hsu PK, Wang BY, Huang CS, Wu YC and Hsu WH: Prognostic factors for post-recurrence survival in esophageal squamous cell carcinoma patients with recurrence after resection. J Gastrointest Surg 15: 558-565, 2011.

11. Ji K, Zhao L, Yang C, Meng M and Wang P: Three-dimensional conformal radiation for esophageal squamous cell carcinoma with involved-field irradiation may deliver considerable doses of incidental nodal irradiation. Radiat Oncol 7: 200, 2012.

12. Gupta T, Agarwal J, Jain S, Phurailatpam R, Kannan S, Ghosh-Laskar S, Murthy V, Budrukkar A, Dinshaw K, Prabhash K, et al: Three-dimensional conformal radiotherapy (3D-CRT) versus intensity modulated radiation therapy (IMRT) in squamous cell carcinoma of the head and neck: A randomized controlled trial. Radiother Oncol 104: 343-348, 2012.
13. Zhang W, Liu X, Xiao Z, Wang L, Zhang H, Chen D, Zhou Z, Feng Q, Hui Z, Liang J, et al: Efficacy of intensity-modulated radiotherapy for resected thoracic esophageal squamous cell carcinoma. Thorac Cancer 6: 597-604, 2015.

14. Jager KJ, van Dijk PC, Zoccali C and Dekker FW: The analysis of survival data: The Kaplan-Meier method. Kidney Int 74: 560-565, 2008.

15. Ni A and Cai J: Tuning parameter selection in Cox proportional hazards model with a diverging number of parameters. Scand Stat Theory Appl 45: 557-570, 2018.

16. Dresner SM and Griffin SM: Pattern of recurrence following radical oesophagectomy with two-field lymphadenectomy. Br J Surg 87: 1426-1433, 2000.

17. Xu Y, Chen Q, Yu X, Zhou X, Zheng X and Mao W: Factors influencing the risk of recurrence in patients with esophageal carcinoma treated with surgery: A single institution analysis consisting of 1002 cases. Oncol Lett 5: 185-190, 2013.

18. Nakamura T, Hayashi K, Ota M, Eguchi R, Ide H, Takasaki K and Mitsuhashi N: Salvage esophagectomy after definitive chemotherapy and radiotherapy for advanced esophageal cancer. Am J Surg 188: 261-266, 2004.

19. Nemoto K, Ariga H, Kakuto Y, Matsushita H, Takeda K, Takahashi C, Takai Y, Yamada S and Hosoi Y: Radiation therapy for loco-regionally recurrent esophageal cancer after surgery. Radiother Oncol 61: 165-168, 2001.

20. Kawamoto T, Nihei K, Sasai K and Karasawa K: Clinical outcomes and prognostic factors of chemoradiotherapy for postoperative lymph node recurrence of esophageal cancer. Jpn J Clin Oncol 48: 259-264, 2018.

21. Liu Q, Cai XW, Wu B, Zhu ZF, Chen HQ and Fu XL: Patterns of failure after radical surgery among patients with thoracic esophageal squamous cell carcinoma: Implications for the clinical target volume design of postoperative radiotherapy. PLoS One 9: e97225, 2014.

22. Ma DY, Tan BX, Liu M, Li XF, Zhou YQ and Lu Y: Concurrent three-dimensional conformal radiotherapy and chemotherapy for postoperative recurrence of mediastinal lymph node metastases in patients with esophageal squamous cell carcinoma: A phase 2 single-institution study. Radiat Oncol 9: 28, 2014.

23. Jingu K, Matsushita H, Takeda K, Umezawa R, Takahashi C, Sugawara T, Kubozono M, Abe K, Tanabe T, Shirata Y, et al: Long-term results of radiotherapy combined with nedaplatin and 5-fluorouracil for postoperative loco-regional recurrent esophageal cancer: Update on a phase II study. BMC Cancer 12: $542,2012$.

24. Miyata H, Yamasaki M, Kurokawa Y, Takiguchi S, Nakajima K, Fujiwara Y, Konishi K, Mori M and Doki Y: Survival factors in patients with recurrence after curative resection of esophageal squamous cell carcinomas. Ann Surg Oncol 18: 3353-3361, 2011.

25. Chen XL, Chen TW, Fang ZJ, Zhang XM, Li ZL, Li H, Tang HJ, Zhou L, Wang D and Zhang Z: Patterns of lymph node recurrence after radical surgery impacting on survival of patients with pT1-3N0M0 thoracic esophageal squamous cell carcinoma. J Korean Med Sci 29: 217-223, 2014.

26. Huang Y, Chen S-W, Fan C-C, Ting L-L, Kuo C-C and Chiou J-F: Clinical parameters for predicting radiation-induced liver disease after intrahepatic reirradiation for hepatocellular carcinoma. Radiat Oncol 11: 89, 2016.

27. Basaki K, Abe Y, Aoki M, Kondo H, Hatayama Y and Nakaji S: Prognostic factors for survival in stage III non-small-cell lung cancer treated with definitive radiation therapy: Impact of tumor volume. Int J Radiat Oncol Biol Phys 64: 449-454, 2006.

28. Zhu D, Ma T, Niu Z, Zheng J, Han A, Zhao S and Yu J: Prognostic significance of metabolic parameters measured by (18)F-fluorodeoxyglucose positron emission tomography/computed tomography in patients with small cell lung cancer. Lung Cancer 73: 332-337, 2011.

This work is licensed under a Creative Commons Attribution-NonCommercial-NoDerivatives 4.0 International (CC BY-NC-ND 4.0) License. 